Rabaska

Revue d'ethnologie de l'Amérique française

\title{
Le Chansonnier manuscrit comme document ethnologique. Considérations sur le cahier de Félix Drouillard (vers 1897-1903)
}

\section{Marcel Bénéteau}

Numéro 1, 2003

URI : https://id.erudit.org/iderudit/201605ar

DOI : https://doi.org/10.7202/201605ar

Aller au sommaire du numéro

Éditeur(s)

Société québécoise d'ethnologie

ISSN

1703-7433 (imprimé)

1916-7350 (numérique)

Découvrir la revue

Citer cet article

Bénéteau, M. (2003). Le Chansonnier manuscrit comme document

ethnologique. Considérations sur le cahier de Félix Drouillard (vers 1897-1903).

Rabaska, (1), 61-78. https://doi.org/10.7202/201605ar d'utilisation que vous pouvez consulter en ligne. 


\section{Le Chansonnier manuscrit comme document ethnologique. Considérations sur le cahier de Félix Drouillard (vers 1897-1903) \\ MarCel BÉNÉTEAU \\ Université de Windsor}

La plupart des chercheurs de chansons folkloriques sur le terrain ont eu l'occasion au cours de leurs enquêtes de prendre connaissance - sinon possession - de documents familiaux reliés au sujet de leur requête : des chansonniers manuscrits dans lesquels étaient consignées les paroles des chansons de grand-père ou de grand-mère, ou d'autres membres de la famille depuis longtemps décédés. Que l'enquêteur reçoive un cahier en consignation pour dépôt éventuel dans un fonds d'archives, qu'il obtienne la permission de le photocopier ou bien qu'il doive se contenter de le parcourir uniquement sur les lieux, les chances sont bonnes que le document demeure aux marges de son enquête. Pour certains, le document écrit se classe nécessairement en deuxième rang après le document oral. L'idée même de paroles écrites semble contraire à la notion que partagent les ethnologues envers la transmission orale. Pour d'autres, la haute fréquence de chansons dites « littéraires » dont témoignent souvent ces cahiers sera cause suffisante pour détourner leur intérêt.

Quoi qu'il en soit, le chansonnier manuscrit demeure une source peu utilisée dans la documentation de la chanson folklorique française. Par exemple, Le Catalogue de la chanson folklorique française de Conrad Laforte, qui répertorie dans ses six volumes plus de 80000 versions de chansons, ne compte qu'environ 25 cahiers manuscrits dans sa bibliographie nord-américaine' D'après les donnés du Répertoire ethnologique de l'Ontario français, la collection du père Germain Lemieux n'inclut aucun cahier manuscrit ${ }^{2}$; cette

1. Le Catalogue de la chanson folklorique française, Québec, Les Presses de l'Université Laval, «Les Archives de folklore »18-23, 1977-1987, 6 vol.

2. Jean-Pierre Pichette, Répertoire ethnologique de l'Ontario français. Guide bibliographique et inventaire archivistique du folklore franco-ontarien, Ottawa, Les Presses de l'Université d'Ottawa, 1992, pp. 185-186. 
même source atteste de la présence sporadique de quelques cahiers dans des collections privées et dans les archives du département d'ethnologie et de folklore de l'Université de Sudbury. Jean-Pierre Pichette a montré l'utilité de cahiers manuscrits comme points de départ et guides pour son enquête chez Donat Paradis, chanteur de Blezard-Valley, dans la région de Sudbury ${ }^{3}$. Monsieur Paradis avait lui-même consigné ses chansons dans trois cahiers ${ }^{4}$.

De toute façon, cette maigre récolte ne représente peut-être qu'une faible proportion d'un véritable gisement de données ethnologiques qui reste à exploiter sous la surface du terrain. Notamment dans la région du Détroit, dans l'extrême Sud-Ouest ontarien, la plupart des familles francophones possèdent ou ont possédé un tel cahier; au cours de nos enquêtes, nous avons eu occasion d'en répertorier une trentaine d'exemplaires. Dans presque autant de familles, d'aucuns regrettent un cahier perdu avec ses centaines de chansons oubliées. À notre avis, ces anciens manuscrits sont d'une valeur inestimable pour l'étude de la chanson traditionnelle. Non seulement servent-ils d'aidemémoire pour les informateurs actuels, leur permettant souvent de retrouver des airs oubliés, mais aussi les meilleurs d'entre eux nous permettent de récupérer le répertoire étendu d'une communauté comme il était constitué à une époque bien antérieure aux enquêtes ethnologiques et aux enregistrements sonores.

Dans les pages qui suivent, nous voulons présenter un cahier qui s'avère de qualité exceptionnelle. En premier lieu, nous allons mettre en évidence son importance dans le contexte de l'étude de la chanson traditionnelle française, non seulement en Ontario, mais à la grandeur de la francophonie. Nous tenterons aussi de répondre à certaines objections quant à la qualité et à l'authenticité des données qu'il contient. En dernier lieu, nous suggérerons quelques pistes à suivre dans une étude éventuelle de ce document.

Le cahier en question a été rédigé par Félix Drouillard, un cultivateur né en 1878 à Rivière-aux-Canards, petit village situé à une dizaine de kilomètres au sud de Windsor. Le manuscrit, relié en carton marbré, porte la date de 1897 sur sa page de titre. Il comprend 176 pages, complètement remplies de chansons disposées en deux colonnes, en plus d'une table des matières. Il y a aussi des paroles consignées sur une dizaine de feuilles volantes insérées à la fin du cahier, dont une qui provient d'un calendrier de l'année 1903 ; il semble donc raisonnable de supposer que le cahier a été rédigé entre cette date et la première mentionnée. Albert Drouillard, fils de Félix, garde toujours en sa possession le manuscrit original ; une photocopie est déposée dans la collection du Centre d'études le Détroit à l'Université de Windsor.

3. Jean-Pierre Pichette, « Coup d'œil sur le répertoire traditionnel d'un chanteur franco-ontarien », Cahiers Charlevoix 2, Sudbury, Société Charlevoix et Prise de parole, 1997, pp. 159-211.

4. Ibid., pp. 162-163. 
Le cahier contient les paroles de 275 chansons, dont 172 chansons traditionnelles, 56 chansons de sources littéraires et 47 chansons anglaises. Pour ajouter à la valeur de ce document déjà si remarquable, monsieur Drouillard identifie la source de chacune des chansons qu'il transcrit ; nous pourrions dire en fait que cet ethnologue avant la lettre note le nom d'une cinquantaine de ses informateurs :

\section{Informateur/trice Nbre de chansons}

Georgine Baron

Rose Beaubien

Armand Cloutier

Anazie Drouillard

Archange Drouillard

Bert Drouillard

Délima Drouillard

Dolphis Drouillard

Edmond Drouillard

Elmire Drouillard

Ferdinand Drouillard

Joséphine Drouillard

Louisa Drouillard

Louise Drouillard

Louisé Drouillard

Michel Drouillard

Nazaire Drouillard

Philomène Drouillard

Sébré Drouillard

François Dufour

Verginie Dufour

Jules Dupuis

Mme Jules Dupuis

Vitaline Girard

Maxime Huneau

\section{Informateur/trice Nbre de chansons}

Eva Laframboise

Eloi Langlois

Adèle Meloche

Elisabeth Meloche

Emily Meloche

Maggy Meloche

Wilfrid Meloche

Emma Monforton

A. (?) Odette

Eli Pageau

Alexina Paré (Drouillard)

Emma Paré

Francis Paré

Mme Joseph Paré

Joséphine Paré

Noé Paré

Élie Renaud

Mary Renaud (Rivière Rouge)

Jennie Ridsdale

Marie Ridsdale

Richard Rocheleau

William Rocheleau

Laumie Tourangeau

Mary Vermette

May Vermette

À quelques exceptions près, la liste des informateurs comprend des noms de familles établies à Rivière-aux-Canards bien avant la fin du XVIII ${ }^{\mathrm{e}}$ siècle ${ }^{5}$.

5. Nous n'avons pas pu identifier Marie et Jennie Ridsdale ; aussi, il n'est pas absolument clair que Louise, Louisa et Louisé Drouillard sont trois personnes différentes. II faudrait entreprendre avant tout une recherche généalogique afin de déterminer les liens de parenté et les relations sociales entre les divers membres de ce groupe. La biographie des informateurs, mettant en valeur leurs origines, occupations, niveaux d'éducation, contacts avec l'extérieur, etc. nous donnerait évidemment des informations fort intéressantes sur la provenance et la diffusion du répertoire. 
Il est évident que la plus grande proportion de chansons vient de la famille Drouillard elle-même (135 chansons ou $49 \%$ ). La famille Paré - dont Alexina Paré, qui épousera éventuellement Félix Drouillard - vivait à Détroit ; on voit donc que la frontière internationale n'était pas un obstacle à la transmission de la tradition orale ${ }^{6}$. Il serait intéressant de retracer la provenance des 57 chansons qui sont manifestement d'origine littéraire : d'après l'orthographe de M. Drouillard, il est évident qu'elles ont aussi été transmises oralement, mais de quelles sources leurs chanteurs les tenaientils ? Les sources des chansons anglaises pourraient aussi nous éclairer sur les transferts culturels entre francophones et anglophones à une époque où la population était encore à forte majorité francophone.

\section{Répertoires traditionnels de la région du Détroit}

Avant d'analyser le contenu du manuscrit Drouillard, il convient de présenter les contextes historique et culturel dans lesquels le cahier a été produit. La majorité des francophones du Sud-Ouest de l'Ontario sont les descendants de deux groupes colonisateurs, arrivés dans la région du Détroit à deux époques distinctes. Ces deux groupes ne se sont pas établis aux mêmes endroits dans la péninsule ; une fois installés, les deux groupes n'ont d'ailleurs pas exploité leur environnement de la même façon. La première vague d'immigrants, comprenant militaires et agriculteurs aussi bien que marchands, artisans et coureurs de bois, commença à occuper les rives de la rivière Détroit à partir de 1701 ; presque tous, directement ou indirectement, étaient dépendants de la traite des fourrures. Certains parmi eux - notamment mais pas exclusivement les militaires - arrivaient directement de la France ; d'autres étaient issus de familles établies dans la vallée du Saint-Laurent au siècle précédent. Cette période de peuplement se prolongea bien au-delà de la fin du Régime français.

En fait, l'immigration de la vallée du Saint-Laurent ne cessa jamais complètement, même après la reddition de Détroit aux Américains en 1796. Mais on peut toutefois affirmer qu'une nouvelle vague d'immigration s'amorce vers 1830, lors d'une crise économique au Bas-Canada, et prend de l'ampleur à partir de 1854 , suite à la construction du premier chemin de fer dans le Sud-Ouest ontarien. Ce deuxième mouvement se poursuit jusqu'au début du $\mathrm{XX}^{\mathrm{e}}$ siècle et aboutit principalement le long du lac Sainte-Claire, à l'est de la ville de Windsor. La grande majorité de ces nouveaux immigrés avaient été

6. Marie Renaud, dont le nom est suivi de la note « Rivière Rouge », serait aussi d'origine américaine ; il en est sans doute de même pour Rose Beaubien, puisque ce patronyme est plutôt typique de la côte nord du Détroit. Il est intéressant de noter que, même de nos jours, l'idée de frontière internationale n'est pas implicite dans la désignation du côté étatsunien de la rivière. Les francophones de la rivière Détroit ne vont pas «à Détroit », « aux États » ou « en Amérique », mais tout simplement « de l'autre bord ». 
cultivateurs au Bas-Canada (plus tard le Québec) et arrivaient dans l'intention de poursuivre le même mode de vie.

Les contacts entre ces deux groupes sont demeurés jusqu'à nos jours relativement restreints. Malgré l'industrialisation générale de la région, les territoires au sud et à l'est de la ville sont restés pendant longtemps essentiellement agricoles et leurs populations très sédentaires ; à Rivièreaux-Canards, certaines familles exploitent toujours le même ruban de terre que leurs ancêtres labouraient au XVIII siècle. Il y a eu relativement peu de mariages entre les deux groupes; chaque région garde ses patronymes typiques. D'après nos recherches, il est clair aussi que chacun des groupes a gardé plusieurs autres spécificités culturelles, particulièrement dans le domaine de la tradition orale. Les répertoires de chansons traditionnelles, entre autres, sont très marquants. Comme nous l'avons démontré ailleurs, les chansons du deuxième groupe se rapprochent de près du répertoire " canadien-français » de la vallée du Saint-Laurent et du Nord de l'Ontario'. Les chansons du premier groupe, par contre, forment un répertoire très distinct, $d$ 'abord en ce qui concerne la typologie, mais aussi du point de vue de la poétique et de la thématique. Sur tous ces points, le répertoire semble avoir plus en commun avec celui recueilli dans les anciens territoires français à l'intérieur du continent $^{8}$.

La mise à jour d'un ancien répertoire de cette région historique est certes d'une importance capitale pour quiconque s'intéresse aux études sur la francophonie du Détroit. Mais le manuscrit Drouillard a des conséquences plus larges pour l'étude de la chanson traditionnelle française en Ontario. Jean-Pierre Pichette, dans son Répertoire, souligne que les références à la chanson traditionnelle française en Ontario avant la fin du XIX ${ }^{e}$ siècle sont

7. Pour une comparaison détaillée des deux répertoires, voir Marcel Bénéteau, Aspects de la tradition orale comme marqueurs d'identité culturelle : le vocabulaire et la chanson traditionnelle des francophones du Détroit, thèse de doctorat, Québec, Université Laval, 2001.

8. Les connaissances au sujet de cet ancien répertoire sont assez vagues et fragmentaires, et une étude approfondie des sources reste à faire. On peut cependant obtenir une idée du caractère et du contenu de ce répertoire du « français du Mid-West » dans quelques œuvres publiées dans la première moitié du XIX' siècle. Voir, par exemple, Cecilia Ray Berry, Folk Songs of Old Vincennes, Chicago, II. T. Fitzsimons Co., 1946 ; Ward Allison Dorrance, The Survival of French in the Old District of Sainte Genevieve, Columbia, The University of Missouri Studies, vol. X, $n^{\circ} .2,1935$. Une collection de chansons recueillies à Vieilles-Mines, au Misssouri, par Joseph-Médard Carrière en 1937 demeure inédite. Thomas Joseph Egan analyse ces sources et en relève d'autres dans sa thèse de doctorat Vincennes, Indiana: echoes of French popular culture, thèse de doctorat, Melbourne, Australie, Monash University, 1990. Pour la synthèse de ce matériel et une première exploration des liens entre la culture du Détroit et celle du Mid-West américain, voir Marcel Bénéteau, «The Oral Traditions of le Détroit and Mid-Western French Settlements: a Missing Link in French Cultural Studies ", dans Le Journal, Centre pour l'étude du pays des Illinois/ Centre for French Colonial Studies, été 2003. 
rares et, pour la plupart, assez obliques ${ }^{9}$. Il relève des témoignages concernant une douzaine de chansons, à partir de commentaires faits par des mémorialistes au XIX ${ }^{\mathrm{e}}$ siècle. Il affirme aussi qu'il y a chez Hubert La Rue et Ernest Gagnon « des généralisations qui nous autorisent à croire que quelques-unes des chansons qu'ils ont publiées avaient cours en Ontario ${ }^{10} \gg$. En plus, certaines chansons publiées par Gagnon seraient de "sources outaouaises », ce qui n'exclut pas la possibilité qu'elles se chantaient sur la rive ontarienne de la rivière. Un témoignage de l'abbé Jean-Baptiste Proulx du lac Abbitibi en 1881 n'est pas plus définitif sur l'appartenance ontarienne des chansons qu'il rapporte ; en réalité, même si on admet que certaines chansons "de voyageurs » puissent avoir été chantées sur la partie ontarienne de ce lac qui chevauche la frontière entre les deux provinces, un répertoire spécifiquement franco-ontarien au XIX ${ }^{\mathrm{e}}$ siècle demeure insaisissable.

Mais la situation au Détroit peut nous éclairer à ce sujet. Plusieurs auteurs britanniques et américains font allusion aux chansons chantées par les habitants français des deux côtés de la rivière aux XVIII ${ }^{e}$ et XIX ${ }^{e}$ siècles ${ }^{11}$. Il faut avouer que ces références sont aussi relativement obliques. Mais trois cahiers manuscrits établissent véritablement les premiers jalons d'un répertoire authentiquement ontarien avant le début $d u X X^{e}$ siècle. Tous les trois proviennent de familles établies dans la région depuis le milieu du XVIII ${ }^{\mathrm{e}}$ siècle. Le premier de ces manuscrits - en effet, un dossier contenant neuf documents déposés au Musée des civilisations (MN PAJ-1-PAJ-9) — provient de la famille Saint-Louis, de Windsor. Les documents semblent avoir été rédigés par Alphonse Saint-Louis (né en 1880) et sa sœur Béatrice (née en 1887) ${ }^{12}$ et portent les dates $1895,1896,1897$ et 1903. Les cahiers de Béatrice semblent être plus ou moins des copies des cahiers de son frère aîné. Ensemble, les documents contiennent 87 chansons, dont 60 de tradition orale française, treize d'origine clairement littéraire et quatorze chansons anglaises.

Un deuxième manuscrit date de 1897 et, comme celui de Félix Drouillard, provient lui aussi de Rivière-aux-Canards. Il s'agit du chansonnier d'Élisabeth Meloche, qui avait 16 ans au moment où elle commençait sa rédaction. Elle écrit également le nom de quelques-uns de ses informateurs, mais moins méticuleusement que Félix Drouillard; sur les 49 chansons françaises du manuscrit, 29 sont attribuées à un chanteur identifié. Nous ne nous attarderons pas davantage sur le contenu de ces deux sources; nous les mentionnons simplement pour confirmer la popularité de la pratique dans la région à cette époque et souligner que le cahier Drouillard s'inscrit dans une tradition déjà

9. Pichette, Répertoire ethnologique, op. cit., p. 22.

10. Loc. cit.

11. Voir Bénéteau, Aspects de la tradition orale, pp. 185-189 pour la discussion de ces sources.

12. Conversation avec Léo Saint-Louis, fils d'Alphonse. 
bien établie. Les données des cahiers Saint-Louis et Meloche (ainsi que celles de quatre cahiers de la famille Monforton, qui datent de 1900 à 1910) ont aussi servi comme points de repères dans l'analyse du cahier Drouillard qui suit.

\section{Aperçu du répertoire traditionnel}

Nous allons nous pencher ici uniquement sur les chansons traditionnelles françaises du cahier Drouillard, en attendant un travail plus approfondi qui traitera également des chansons littéraires et anglaises. Pour les besoins de l'analyse, nous nous servons de la classification développée par Conrad Laforte pour son Catalogue. Le répertoire traditionnel se divise en six catégories basées sur la structure poétique des chansons : chansons en laisse, chansons strophiques, chansons en forme de dialogue, chansons énumératives, chansons brèves et chansons sur les timbres. Le cahier comprend 129 chansons types identifiées par Laforte. Nous considérons aussi comme «traditionnelles » 43 chansons non classées par Laforte mais qui sont clairement de tradition orale.

La composante traditionnelle du répertoire Drouillard est particulièrement digne de notre attention. On y retrouve de nombreuses chansons qui sont des attestations uniques en Ontario français et d'autres qui sont complètement inconnues en Amérique francophone. La grande majorité sont des chansons strophiques (132, ou $77 \%$ du total). La deuxième catégorie en importance est celle des chansons en forme de dialogue : 22 chansons, ou $13 \%$; à elles seules, ces deux formes narratives représentent $90 \%$ du répertoire traditionnel. On n'y retrouve que six chansons énumératives, cinq chansons sur les timbres et sept chansons en laisse. La part de cette dernière catégorie est quelque peu déconcertante ( $4 \%$ du répertoire traditionnel), si l'on considère la place qu'occupe la chanson en laisse dans la perception populaire du répertoire canadien-français (environ $35 \%$, d'après ce que Jean-Pierre Pichette appelle le répertoire « laurentien $\rangle^{13}$ ). L'absence de chansons de cette catégorie est encore plus étonnante si on tient compte du fait que, parmi les textes que Félix Drouillard inclut dans son cahier, on retrouve toutes les chansons les mieux connues à Rivière-aux-Canards; dans certains cas (Pierre et Françoise, et Les Amants séparés par le père), il inclut même deux variantes de la même chanson. Rien alors ne nous porte à croire que monsieur Drouillard avait l'intention de rédiger autre chose que le répertoire complet de son milieu familial ; ce n'était clairement pas son but de présenter une « sélection » de chansons. Nous reviendrons sur cette question des chansons en laisse plus tard dans l'article.

13. Jean-Pierre Pichette, « Coup d'œil sur le répertoire... », pp. 177-179. 
Parmi ses nombreuses richesses, le manuscrit comprend 37 chansons types qui ne sont pas attestées ailleurs en Ontario, d'après le Catalogue Laforte. Nous en dressons la liste complète ci-dessous (la cote et le titre sont ceux du Catalogue):

\section{Chansons strophiques}

C7 La Sœur mise à l'épreuve

C29 Le Passage de l'eau : les îles

C47 La Fille égarée dans un bois : la meunière

C49 Le Rosier de trois couleurs de roses (et ?)

D28 Le Matamore

E1 L'Amant malheureux (2 versions)

E12 L'Amant fidèle et le vin

E26 La Belle, je n'ai point d'autre amour que vous

E45 Le Galant et les filles difficiles

E50 La Maîtresse si belle

F17 Le Galant refusé : le père n'a pas assez d'argent

F22 Le Berger repoussé par la belle bergère

F35 La Bergère, son amant et le vieillard

H16 Départ du marin

H34 La Californie, le climat de

$\mathrm{H} 47$ Loin de sa mère

I24 Départ et retour de l'amant fidèle

L58 Le Retour des chantiers la blonde mariée

L60 Le Retour du voyageur

O30 La Fille sans amant
P2 Le Jour de mes noces

P24 Les Amants séparés par le père

Q7 L'Ivrogne content de sa femme

R7 Conseils aux garçons

\section{Chansons en laisse}

\section{A7 Le Martyre de sainte Catherine}

\section{Chansons en forme de dialogue}

A6 Galant, tu perds ton temps

A11 Sommeilles-tu, ma petite Louison

A22 L'Anneau d'or

B3 Le Berger et la bergère

B4 Le Berger infidèle

$\mathrm{B} 10$ La Bergère infidèle et son berger

B11 Bergère, goûtons au plaisir de l'amour

B15 La Fille égarée dans un bois et le monsieur

C9 Je connais mon affaire

C14 Mariez-moi, sinon je ne filerai pas

\section{Chansons énumératives}

$\mathrm{Ha} 7$ Le Mari que je voudrais

\section{Chansons sur des timbres}

C8. 54. 1 Tintin

Les versions du manuscrit Drouillard sont parfois très différentes des versions recueillies ailleurs ; certaines d'entre elles témoignent aussi de voies de transmission fort mystérieuses. Par exemple, la chanson strophique Le Rosier de trois couleurs de roses est bien connue dans l'est du Canada; Laforte en recense treize versions, dont dix du Nouveau-Brunswick. Une seule version, recueillie par Archange Godbout ${ }^{14}$, qui a effectivement comme incipit « C'est au détroit de ces beaux jours » met en valeur un scénario semblable à la version du manuscrit Drouillard. Les deux premières strophes sont communes à toutes les versions du Catalogue; par la suite la plupart mettent en valeur un scénario dans lequel la belle porte dans sa main « un rosier de trois couleurs de roses ». Seules les versions de Godbout et de Drouillard se déroulent de la façon suivante :

14. Chantée par M. Wilbrod Papillon des Écureuils (Portneuf), Archives de folklore de l'Université Laval, collection Archange Godbout, ms. $n^{\circ} 20$. 


\section{VOICI LE CARÊME}

chantée par M. Wilbrod Papillon les Écureuils (Portneuf), vers 1918-1920 coll. Archange Godbout, ms. $n^{\circ} 20$

C'est au détroit de ces beaux jours, Lui a trois jolies filles, Lui en a une que j'aime surtout, Car il y a bien longtemps que je lui fais l'amour.

Un soir, en allant pour la voir, Son petit cœur soupire :

—Mais qu'a-vous, belle, à tant soupirer? Je suis venu, ce soir, c'est pour vous reconsoler.

- Monsieur, ça ne dépend pas de moi, Ça dépend de ma mère Ma mère aussi de tous mes parents, Car il y en a aucun qu'ils en sont consentants.

\section{C'EST DANS LES ÎLES DE SAINT-THOMAS}

ms. Félix Drouillard, p. 59

Rivière-aux-Canards, vers 1897

chanson de sa sceur, Délima Drouillard

coll. MB 208. 04a

C'est dans les îles de Saint-Thomas,

Lui a des jolies filles.

Lui en a une qui est par-dessus tout

La celle que mon cœur

lui fait l'amour.

Le soir quand je vas la voir chez-elle, Son petit cour soupire.

- Ah, qu'av'-vous belle, à tant soupirer? Je suis venu pour vous consoler.

- Galant, ça revient pas de toi, S'est revient de mon père.

Ah ni mon père, ah ni mes parents Lui en a aucun qui sont consentants.

- Content, content, seront contents, Ma très chère maîtresse.

- Content, content, seront contents, Tu seras toujours mon fidèle amant.

Ah, c'est les garçons du Détroit, Préparez vos carrioles.

Entertenez vos chevaux bien gras

Pour promener les filles jusqu'au mardi-gras.

Voilà le carême qui est commencé, Nous faudra faire pénitence.

À la santé de ce bon repas, Nous faudra manger des fèves et des pois.

La chanson en forme de dialogue Le Berger et la bergère représente un cas particulièrement intrigant. Une seule version figure au Catalogue Laforte, chantée en 1917 par V. -F. de Repentigny. Notre version, du manuscrit Drouillard, comprend cinq strophes de six vers; nous notons que les deux derniers vers de la quatrième strophe sont absents. Curieusement, les deux 
mêmes vers sont absents de la version de monsieur de Repentigny, indiquant un lien indéniable, mais jusqu'à présent indéterminé, entre les deux versions ${ }^{15}$.

\section{INGRATE BEAUTÉ, INSENSIBLE BERGÈRE}

chantée par Vincent-Ferrier de Repentigny

É. -Z. Massicote,

Journal of American Folklore

Vol. $32, \mathrm{n}^{\circ} 123,1919$, pp. $46-48$

- Va, ingrate beauté, insensible bergère ! $\mathrm{Qu}[\mathrm{e}$ t'ai-je] donc fait pour me traiter ainsi?

Je ne te vois plus sur la verte fougère.

Tu pousses mon cour à des cruels soupirs.

Reviens à moi ;

je suis tendre et sincère ;

Prends donc pitié d'un amoureux transi.

Je t'ai bien cueilli, Jeanneton, sur la ruette,

Des fleurs nouvell(es), pour te faire un bouquet.

J'avais d'un beau ruban couronné ma [c]ouette,

Pour te fair [e] par $[\mathrm{t}]$ de ce beau bouquet.

$\mathrm{Tu}$ as réjoui de vertes retraites ;

Tu as joué dessur mon clair jalet.

J'ai bien gardé tes moutons, sur la ruette,

Dans les plus grand[es] chaleurs de l'été.

J'avais des beaux ormeaux qui formaient bel ombrage,

Pour te garantir de l'ardeur du soleil,

Me promenant $d u$ long du vert bocage

En attendant l'air de ton réveil.

J'ai (é)té te chercher de l'eau d'une fontaine,

Que j'ai [ap]portée pour te désaltérer.

J'ai tant couru, j'en ai tant perdu l'haleine, $\mathrm{Ah} ! \mathrm{Va}$, ingrat(e), tu ris de mes bontés.

\section{DIS-MOI, CRUELLE INSENSIBLE BERGÈRE}

ms. Félix Drouillard, pp. 87-88

Rivière-aux-Canards, vers 1897

chanson de Délima Drouillard

coll. MB 202. 19a

- Dis-moi, cruelle insensible bergère,

Que t'ai-je donc fait pour me quitter ici?

Je ne t'y vois plus sur la verte fugère.

Tu plonges mon cœur dans un cruel soupir.

Reviens à moi, je suis tendre et sincère,

Ah, prends pitié d'un amour intrancé.

J'ai été garder les moutons sur l'harbette

Pendant les plus grandes chaleurs de l'été.

Sous ces ormeaux, formant une belle ombrage.

Là où je vais chez mon père, où j'allais Pour me garantir de l'ardeur du soleil, Pour me réjoui dessur ces verts bocages.

J'ai été cueillir dessur la tendre harbette

Des fleurs nouvelles pour toi, faire un bouquet.

Avé[c] du ruban, j'ai armé ta houlette, Je t'en ferai part de ce beau bouquet

Pour te réjouir dans cette belle retraite,

Là où je vais sur mon père, où j'allais.

J'ai été chercher de l'eau à la fontaine, Que je t'emportai pour te désaltérer.

J'ai tant couru, j'en ai perdu haleine ; Mon beau berger, tu ris de mes bontés.

15. D'après la biographie sommaire de Repentigny publiée par Barbeau (dans The Journal of American Folklore, vol. 32, $\mathrm{n}^{\circ}$ 123, janv.-mars 1919, pp. 46-48), nous savons que le chanteur passa par les chantiers forestiers de Muskegan, au Michigan, en 1887. Aurait-il rencontré quelqu'un du Détroit à ce moment, lui transmettant la version unique de cette chanson en Amérique du Nord ? Les vers absents sont-ils restés en France (où la chanson a disparu complètement), ou se sont-ils perdus quelque part entre Beauharnois et le Détroit? 
$[\cdots$

[

— Tu te plains à tort,

beau berger que j'adore,

Car de trop aimer

c'est dans mon tendre cœur.

Toute la nuit je dors ni je sommeille ;

Je pense à toi en attendant le jour.

- Ah, tu te plains à tort, belle bergère que j'adore,

C'est de trop aimer, de mon tendre cœur.

Je t'ai aimé, ah oui ! Et je t'aime encore. Je t'ai aimé, je t'aime bien encore, Reviens, berger, je t'aimerai toujours. Mon beau berger, je t'aimerai toujours.

Plusieurs chansons non classées au Catalogue Laforte sont aussi d'un intérêt particulier. Certaines figurent dans des fonds d'archives partout en Amérique française; d'autres ne sont connues qu'en Europe. Par exemple, une version unique de la chanson suivante, recueillie par Victor Smith en Velay et Forez au XIX ${ }^{e}$ siècle, est répertoriée dans le Catalogue Coirault sous le titre La petite Allemande $\left(\mathrm{n}^{\circ} 1214\right)$. La chanson semble être inconnue en Amérique du Nord, sauf pour la version du manuscrit Drouillard :

\section{LA PETITE ALLEMANDE}

manuscrit de Félix Drouillard, pp. 158-159

Rivière-aux-Canards, vers 1897

chanson d'Adèle Meloche

Coll. MB 208. 40a

- Je viens en ce jour

Te faire l'amour,

Ma petite Allemande.

Je viens en ce jour

T'y faire la court [sic],

Pour t'y parler d'amour.

Je viens sincèrement

A l'annonce du roi,

D'une amitié sincère,

Et ton cœur

Je ferai ton vrai serviteur. (bis)

- Na ni, mon cher cœur,

Je suis fille d'honneur,

Cherchez une autre Allemande.

$\mathrm{Na}$ ni, mon cher cœur,

Je suis fille d'honneur,

Cherchez une autre ailleurs.

Je viens du moulin,

De faire moudre mon grain,

En attendant le rolle [?]

Vous savez, ça me convient pas

De parler avec un soldat. (bis)

- Na ni, mon cher cœur,

De moi n'aies point peur,

Tu me feras ma femme.

$\mathrm{Na}$ ni, mon cher cœur,

De moi n'aies point peur,

Je serai ton serviteur.

Car dans six mois d'ici,

Je serai le Français,

J'aurai la joie dans l'âme.

Je serai du jour et de nuit

Au bel [bal ?] et à la comédise. (bis)

- Si je savais cela,

Je poursuivrais vos pas

Partout dedans la France.

Si je savais cela,

Je poursuivrais vos pas,

Parlera qui voudra.

Car dans six mois,

Je serai dans Paris,

Je viverai comme une R[eine ?].

J'estimerais m'en aller avec vous

Que rester à planter des choux. (bis) 
Les cahiers manuscrits représentent-ils authentiquement le répertoire régional ?

Nous avons proposé que les cahiers manuscrits contiennent des données qui sont indispensables pour le chercheur qui veut reconstituer l'ancien répertoire d'une région déterminée et analyser le contexte culturel dans lequel celui-ci s'est élaboré. Mais il faut d'abord examiner la question de la qualité et de l'authenticité des données du manuscrit.

Les cahiers manuscrits soulèvent en fait deux questions. Nous pouvons répondre assez rapidement à la première, à savoir : puisque la chanson y est forcément donnée sous une forme écrite, quelle est sa valeur dans une étude qui porte sur la «tradition orale" ? Nous pouvons dire que les cahiers représentent sans aucun doute la tradition orale à un moment déterminé. L'orthographe non normalisée — parfois phonétique — du rédacteur nous assure déjà que les chansons n'ont pas été copiées de sources écrites. Ces manuscrits ne sont certes pas destinés à un public savant ou lettré — c'est-àdire à la lecture - mais servent plutôt à faciliter l'exécution orale des chansons. Dans le passé comme aujourd'hui, les cahiers avaient la fonction d'aide-mémoire; lors d'une conversation récente avec la bru d'Élisabeth Meloche, celle-ci nous affirma que « le cahier faisait le tour de la chambre avec le verre puis la bouteille ${ }^{16}$.

La deuxième question est plus complexe : comment pouvons-nous déterminer les critères de sélection des rédacteurs des cahiers ? En d'autres termes, comment nous assurer que les cahiers reflètent tous les volets de la chanson traditionnelle présente dans un répertoire régional ? Le problème se manifeste avant tout autour de la question des chansons en laisse, et c'est sur cet aspect que nous allons maintenant revenir.

L'absence quasi totale de la chanson en laisse dans certains cahiers remonte au cœur du problème : n'indiquerait-elle pas de sérieuses lacunes dans la représentation du répertoire local ? Rappelons les proportions du manuscrit Drouillard : sur les 172 chansons qui constituent le répertoire traditionnel, seulement sept (4\%) sont des chansons en laisse. Étant donné l'ancienneté de cette catégorie et la position privilégiée qu'elle occupe dans la plupart des collections canadiennes-françaises, on serait porté à croire que ces proportions reflètent fautivement la réalité objective ; d'autant plus si l'on tient compte de l'ancienneté du peuplement du Détroit et de l'apport plus que probable des voyageurs et coureurs de bois à son répertoire.

Il faut donc tenter de comprendre si, et pourquoi, un compilateur méticuleux comme Félix Drouillard aurait exclu une partie majeure du

16. Conversation avec Anna Bastien, 90 ans, le 28 février 2003. 
répertoire local de son cahier de chansons. Trois explications sont possibles. Tout d'abord, et en cela nous partageons en partie l'opinion de Lucien Ouellet du Musée canadien des civilisations : les gens mettaient en général dans leurs cahiers les chansons qu'ils entendaient rarement, afin de ne pas les oublier ${ }^{17}$. C'est sûrement le cas pour certains cahiers provenant d'autres régions du Canada français que nous avons eu l'occasion d'examiner, cahiers qui contiennent presque exclusivement des chansons littéraires. D'après ce point de vue, on n'avait pas besoin d'inscrire les chansons en laisse dans les cahiers, puisque tout le monde les connaissait. La forme même des chansons en laisse les rend aptes à être facilement rappelées ; alors pourquoi utiliser l'espace limité dans un cahier en copiant des chansons qui s'apprennent toutes seules? Il valait bien mieux réserver la place aux belles chansons rarement entendues et difficilement retenues.

Mais l'analyse du manuscrit Drouillard, ainsi que de la plupart des autres cahiers à la rivière Détroit, indique qu'on y inscrivait en fait même les chansons les mieux connues du répertoire - et ce dans toutes les catégories. Là-haut sur ces montagnes (II. F-5), La Belle qui entre au couvent (II. F-12), Prendre un petit coup c'est agréable (II. R-16), L'Ivrogne grondé par sa femme jalouse (III. F-4), La Destinée, la rose au bois (I. M-6) : ce sont toutes des chansons qui figurent communément dans les cahiers de la rivière Détroit, bien qu'elles se trouvent aussi parmi les pièces les plus fréquentes du répertoire actuel. Comme nous l'avons vu, monsieur Drouillard inclut même parfois deux versions de la même chanson; effectivement, pour lui, il ne semble pas y avoir de chansons « trop bien connues ». En plus, nous savons que la grande majorité des informateurs de monsieur Drouillard était des membres de sa famille ou de sa communauté immédiate ; on n'a donc pas affaire ici à des chansons entendues au hasard lors d'un voyage ou chantées par un passant étranger. Ce sont sans doute des chansons entendues à maintes reprises, lors de soirées et de fêtes familiales.

Il est vrai que parmi les sept laisses copiées dans son cahier, Félix Drouillard en inclut trois qui sont rares à la rivière Détroit : Le Martyre de Sainte Catherine, La Danseuse noyée, et Mon père a fait bâtir maison sur le bout d'un pont sont effectivement des versions uniques dans la région. Mais les quatre autres laisses sont très bien connues à la rivière Détroit, et nous en avons recueilli des versions multiples : La Destinée, la rose au bois, La Rose blanche, un croisement particulier de La Fille au cresson et de La Bergère et le vieillard, et La Demande en mariage refusée. De plus, la majorité des autres laisses populaires sont représentées sporadiquement dans d'autres

17. Conversation personnelle, le 17 mars 1994. 
cahiers de la région (À la claire fontaine, Le Bateau chargé de blé, Ivrogne : femme à vendre, etc.).

Une deuxième explication ${ }^{18}$ a trait à la forme même de la chanson en laisse. Certaines formules strophiques (parmi les 61 analysées par Laforte) sont très complexes, avec des répétitions de vers et des refrains intercalés en plusieurs parties. Avant la publication de l'analyse de Laforte, même les experts ne s'entendaient pas toujours sur la façon de transcrire de telles chansons ; il n'est donc pas déraisonnable de supposer qu'un agriculteur quasi analphabète hésiterait à mettre sur papier de tels vire-langues. Mais en examinant le répertoire des laisses recueillies sur le terrain de notre enquête, on se rend compte que la forme chantée de la plupart de ces chansons demeure relativement simple. Les refrains à grande complexité ne sont pas une des caractéristiques de la chanson en laisse du Sud-Ouest ontarien. Mais lorsqu'une des rares chansons avec un tel refrain se présente dans le répertoire - à savoir, une version de Mon père a fait bâtir maison sur l'bout d'un pont - Félix Drouillard se montre parfaitement à la hauteur de la situation. On constate que le jeune rédacteur saisit bien la forme de la chanson, à tel point qu'il se permet d'employer certains raccourcis dans sa transcription. Rien n'indique qu'une forme complexe l'aurait découragé à transcrire d'autres chansons en laisse. Nous reproduisons la chanson telle qu'elle paraît dans le cahier manuscrit (nous avons cependant ajouté les caractères gras et italiques pour faciliter la lecture $)^{19}$ :

\section{No 223 Premier Couplet}

Mon père a fait bâtir maison

Vivre lamourette enver jaton

La fait bâtir sur le bout dun pont -

\section{Chorus}

Sur le vit sur le vin sur la St-Henry

Sur la queue du rum

Belle rum jolie rum flit-flan flan

Sur la mer Imaculée

Vivre lamourette enver jaton

Malurie dondis

\section{Deuxième Couplet}

Son trois charpentier quil la font Vivre lamourette enver jaton A mon père faite moi un donc sur vit sur vin

\section{Troisième Couplet}

A mon père faite moi un donc Vivre lamour enver jaton

De me donner votre maison sur vit -

18. Cette deuxième explication ressort aussi de notre conversation avec Lucien Ouellet. 19. Manuscrit Félix Drouillard, p. 137. 
Quatrième Couplet

De me donner votre maison

Vivre lamourette enver jaton

A ma fille prometter moi donc sur vit -

\section{Cinquième Couplet}

A ma fille prometter moi donc

Vivre lamourette enver jaton

De jamais aimer les garçon

sur le vit -

\section{Sixième Couplet}

De jamais aimer les garçon

Vivre lamourette enver jaton

Jestimerais mieux que la maison

\section{Septième Couplet}

Jestimerais mieux que la maison

Vivre lamourette enver jaton

Quelle soit en cendre et en

charbon sur le vit -
Septième Couplet [sic]

Quel soit en cendres et en charbon Vivre lamourette enver jaton

Vous vous seriez sur le pignons

Huitième Couplet

Vous vous seriez sur le pignons

Vivre lamourette enver jaton

Vous vous chauferier les talon

Neuvième Couplet

Vous vous chauferier les talon

Vivre lamourette enver jaton

Aussi la barbe et le mâton

Dixième Couplet

Aussi la barbe et le mâton

Vivre lamourette enver jaton

Vous vous chauferie tous du long

Archange Drouillard

Reste encore une troisième raison qui peut expliquer l'absence de chansons en laisse dans les cahiers manuscrits, et c'est celle-là qui nous paraît la plus évidente : la chanson en laisse occupe tout simplement une place moins importante dans le répertoire de la rivière Détroit qu'ailleurs au Canada français. On retrouve une situation analogue dans les répertoires d'autres communautés francophones isolées, comme par exemple chez les Cajuns de la Louisiane, les Métis de l'Ouest canadien et les anciens peuplements francophones du Mid-West américain. Nous avons suggéré ailleurs ${ }^{20}$ que la popularité de la chanson en laisse est un phénomène qui se fait remarquer d'abord dans les premières publications de chansons canadiennes-françaises au XIX $X^{c}$ siècle et qui s'intensifie au XX' siècle grâce au rôle des médias basés à Québec, Montréal et Ottawa ; la vogue de la « chanson à répondre » doit aussi sans doute beaucoup au travail dans les chantiers, un autre phénomène 


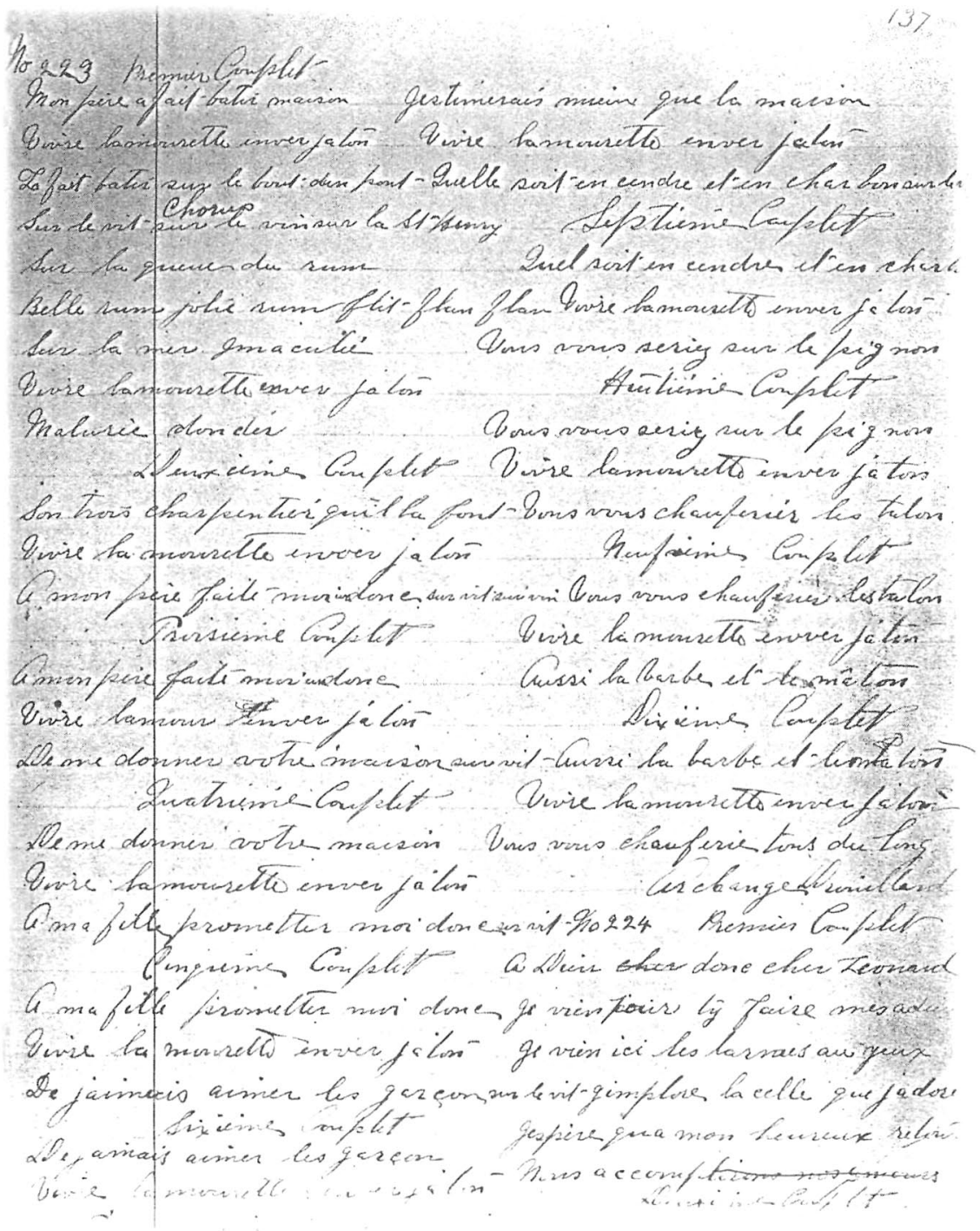

«Mon père a fait bâtir maison »

Manuscrit Félix Drouillard 
qui ne fut jamais un facteur important pour la population francophone établie à la rivière Détroit. Ces observations sont appuyées par le fait que même chez le deuxième groupe francophone établi au lac Sainte-Claire vers la fin du XIX ${ }^{\mathrm{e}}$ siècle - un groupe beaucoup plus proche de ses origines québécoises - la chanson en laisse occupe une place beaucoup plus importante. Toutes sources confondues, les chansons en laisse composent environ $13 \% \mathrm{du}$ répertoire chanté par les francophones de la rivière Détroit ; par contre, chez le groupe avoisinant établi au lac Sainte-Claire, le pourcentage s'élève à $26 \%$. Les témoignages recueillis du côté américain du Détroit par des chercheurs comme Dennis $\mathrm{Au}$ et Neil Johnson sont particulièrement révélateurs à ce sujet, puisque les sources manuscrites sont inexistantes chez cette population qui n'a jamais su ni lire ni écrire le français. Sur un répertoire de 44 chansons recueillies par les deux chercheurs, nous ne relevons que trois chansons en laisse (environ $7 \%$ ) $^{21}$. Il est significatif que l'un des informateurs principaux de Dennis $\mathrm{Au}$, le père Lambert LaVoy, affirme même n'avoir jamais entendu la chanson Trois beaux canards quand il était jeune ; d'après lui, il s'agit d'une chanson du Québec et il insiste : «those weren 't our songs ${ }^{22}$ ».

Donc, sans écarter entièrement l'effet des deux premiers facteurs chansons trop bien connues et la difficulté de la transcription - il nous semble fort probable que les cahiers manuscrits recueillis dans les communautés de la rivière Détroit - dont celui de Félix Drouillard - représentent d'assez près le répertoire chanté dans cette région. La valeur de ce cahier comme artefact culturel est donc d'autant plus important.

\section{Vers une étude globale du manuscrit Drouillard}

Le chansonnier manuscrit de Félix Drouillard, effectivement un des premiers documents ethnologiques de l'Ontario français, suggère une multitude de sujets à exploiter dans une étude éventuelle. Le document nous fournit un portrait culturel tout à fait inattendu d'une petite communauté francophone - effectivement, la plus ancienne en Ontario, et aujourd'hui l'une des plus assimilées - au tournant du $\mathrm{XX}^{\mathrm{e}}$ siècle, au tout début de l'influence anglaise. Le fait que les informateurs soient identifiés nous permettra de retracer les liens de parenté et de proximité entre eux ; l'élaboration de leurs occupations, de leurs contacts avec l'extérieur, de leurs réseaux de connaissances nous fourniront sans doute de nouvelles perspectives sur la provenance et la transmission d'un répertoire régional. Qui contribue à un répertoire et dans quelle mesure ? Quel est le rôle et l'apport des chansons dites « littéraires »,

21. Collection Dennis M. Au, cassettes $n^{o s} 1-8$, Monroe et Erie, Michigan, 1975-1986, collection privée, copies déposées au Centre d'études le Détroit ; collection Neil Johnson, Mt. Clements, Michigan, 1961, Wayne State University Folklore Archives, Détroit ; copie déposée au Centre d'études le Détroit.

22. Lambert LaVoy, coll. Dennis M. Au, nº 8, le 7 septembre 1976. 
jusqu'à présent largement mises de côté dans l'étude de la tradition orale? Quelle est leur provenance ? Comment s'insèrent-elles dans la thématique du répertoire folklorique? Les chansons anglaises doivent-elles être considérées comme éléments étrangers à la culture, ou se conforment-elles aux niveaux de la forme et du contenu au répertoire déjà présent dans la communauté ? Quelle proportion de ce répertoire historique se maintient sur le terrain d'aujourd'hui?

Il y a matière, dans de telles problématiques, à intéresser les historiens et les sociologues autant que les ethnologues. L'orthographe quasi-phonétique utilisée dans la transcription pourrait aussi donner lieu à des études linguistiques fort pertinentes. Une édition diplomatique et commentée du manuscrit de Félix Drouillard, en plus d'éclairer un terrain peu étudié, pourrait aussi suggérer de nouvelles approches pour un retour sur des terrains déjà bien sillonnés par les enquêteurs. 